\title{
Bias from network misspecification under spatial dependence*
}

\author{
$\operatorname{Timm~Betz~}^{\dagger}$ \\ Scott J. Cook \\ Florian M. Hollenbach ${ }^{\S}$
}

\begin{abstract}
The pre-specification of the network is one of the biggest hurdles for applied researchers in undertaking spatial analysis. In this letter, we demonstrate two results. First, we derive bounds for the bias in non-spatial models with omitted spatially-lagged predictors or outcomes. These bias expressions can be obtained without prior knowledge of the network, and are more informative than familiar omitted variable bias formulas. Second, we derive bounds for the bias in spatial econometric models with non-differential error in the specification of the weights matrix. Under these conditions, we demonstrate that an omitted spatial input is the limit condition of including a misspecificed spatial weights matrix. Simulated experiments further demonstrate that spatial models with a misspecified weights matrix weakly dominate non-spatial models. Our results imply that, where cross-sectional dependence is presumed, researchers should pursue spatial analysis even with limited information on network ties.
\end{abstract}

Key Words: spatial dependence, bounds, omitted variables, measurement error

\author{
Conditionally Accepted at Political Analysis
}

\footnotetext{
*An earlier version of this paper benefited from feedback at the 2017 Annual Meeting for the Society of Political Methodology. We particularly thank Andrew Bridy, three anonymous reviewers, and the editor for their useful advice. All remaining errors are ours alone. Authors are listed in alphabetical order, equal authorship is implied. For further questions contact Scott J. Cook. Portions of this research were conducted with high performance research computing resources provided by Texas A\&M University (http://hprc. tamu. edu). The replication materials for this paper can be found at Hollenbach, Betz and Cook (2019).

${ }^{\dagger}$ Assistant Professor of Political Science, Department of Political Science, Texas A\&M University, College Station, TX 77843. Email: timm.betz@tamu.edu, URL:www.people.tamu.edu/ timm.betz

${ }^{\ddagger}$ Assistant Professor of Political Science, Department of Political Science, Texas A\&M University, College Station, TX 77843. Email: sjcook@tamu.edu, URL: scott jcook.net

${ }^{\S}$ Assistant Professor of Political Science, Department of Political Science, Texas A\&M University, College Station, TX 77843. Email: fhollenbach@tamu.edu, URL: fhollenbach.org
} 
Across the social sciences, theories involve cross-unit interactions resulting from spillovers in predictors (e.g., externalities) or outcomes (e.g., interdependence). Where researchers are explicitly interested in cross-unit relationships, spatial econometric models are widely used (Anselin, 1988; Franzese and Hays, 2007). Even if researchers are otherwise uninterested in these relationships, however, accounting for spatial dependence is often necessary to recover unbiased estimates.

With a variety of spatial models to select from and widely-available software routines, estimating spatial models is easier than ever. Yet, one prerequisite for spatial analysis continues to frustrate applied researchers: the specification of the spatial weights matrix. Specifying the spatial weights matrix requires additional theories and data for cross-unit relations (Neumayer and Plümper, 2016). Researchers, however, often lack theory-backed information to motivate this choice (Corrado and Fingleton, 2012). As a result, any spatial weights matrix can be contested and the value of the resulting estimates disputed. This may lead researchers away from spatial econometric models and toward models that ignore spatial relationships - especially when understanding spatial relationships is not the primary concern.

In this note, we consider the consequences from ignoring spatial interactions outright and from introducing them with error in the weights matrix. We first derive bounds for the bias from ignoring spatial dependence. Exploiting several features unique to spatial relationships, we obtain bounds that are more informative than common expressions for omitted variables bias. We then demonstrate that omitting spatial terms produces worse results than estimation based on a misspecified network under non-differential error. As such, we argue that researchers should prefer spatial models, even when they possess limited knowledge of the network.

\section{Confounding from omitted spatial dependence}

We consider two spatial processes: spillovers of predictors across units, in the form of a spatial lag of X (SLX) model; 2) outcome interdependence between units, in the form of a spatial autoregressive (SAR) model. 


\section{Consider a SLX data-generating process:}

$$
\mathbf{y}=\alpha+\mathbf{x} \beta+\mathbf{W} \mathbf{x} \theta+\epsilon
$$

where $\mathbf{y}, \mathbf{x}$, and $\epsilon$ are $N$-length vectors of the outcome, predictor, and error term, respectively. W is an $N$-by- $N$ spatial weights matrix specifying network ties between units. We make usual assumptions about $\mathbf{W}$ : it has zeroes along the diagonal, non-negative elements, and is normalized using row-standardization or min-max normalization. Interest is in estimating the coefficient $\beta$.

Omitting $\mathbf{W} \mathbf{x}$ results in standard omitted variables bias:

$$
\operatorname{plim}_{n \rightarrow \infty} \hat{\beta}_{O L S}-\beta=\theta \frac{\operatorname{cov}(\mathbf{W} \mathbf{x}, \mathbf{x})}{\operatorname{var}(\mathbf{x})}
$$

Our goal is to identify, for given sample realizations of $\mathbf{W}$ and $\mathbf{x}$, more informative bounds for the bias than expression (2). ${ }^{1}$ Usually, the omitted variable bias formula does not offer much leverage, because the covariances involving the omitted terms are unknown. When the omitted variable is a spatial lag of a predictor, however, we have more information, because $\mathbf{W x}$ is a linear combination of the values of $\mathrm{x}$. Consequently, knowledge of $\mathrm{x}$ is sufficient to produce empirical bounds.

Specifically, for a large class of common weights matrices, ${ }^{2}$

$$
\left|\frac{\operatorname{cov}(\mathbf{W} \mathbf{x}, \mathbf{x})}{\operatorname{var}(\mathbf{x})}\right| \leq 1
$$

Under these conditions, the upper bound of the bias in equation (2) is $\theta$. In many contexts, ownunit values of a predictor can be reasonably assumed to have a larger effect than other-unit values,

\footnotetext{
${ }^{1}$ The standard errors are also biased (Franzese and Hays, 2007). A similar approach to ours may extend to the variance-covariance matrix, which we leave to future work.

${ }^{2}$ We provide full results in the Appendix. For intuition, note that $\mathbf{W}$ often induces averaging of the values of the original vector $\mathbf{x}$, thereby reducing the variance. A sufficient condition for inequality (3) to hold is that Moran's I in the sample is bounded by $[-1,1]$, which generally holds except "for an irregular pattern" (Cliff and Ord, 1981). We demonstrate that this inequality holds for any symmetric weights matrix; for any spectral weights matrix; and for any doubly-stochastic weights matrix. This includes all matrices based on the attributes of undirected dyads, such as inverse distance, bilateral flows, threshold models, and contiguity. For arbitrary combinations of $\mathbf{W}$ and $\mathbf{x}$ the inequality may no longer hold. We derive worst-case bounds for arbitrary $\mathbf{W}$ that can be calculated from the sample, and show that inequality (3) holds for any arbitrary weights matrix when $\mathbf{x}$ is binary.
} 
such that $|\beta| \geq|\theta|$. This implies that the maximum asymptotic bias is $\beta .^{3}$

Thus, for any given sample, $\beta$ provides an upper bound on the bias in $\hat{\beta}_{O L S}$, and, asymptotically, $\hat{\beta}_{O L S}$ is in the interval $[0,2 \beta]$. Except for randomness, therefore, we should not observe sign switches as a consequence of omitting Wx. Moreover, given a potentially biased estimate $\hat{\beta}_{O L S}$, we can estimate the lower bound of $\beta$ as $\frac{\hat{\beta}_{O L S}}{2}$. This lower bound shifts closer toward $\hat{\beta}_{O L S}$ as the magnitude of spillovers decreases, allowing for assessment of the sensitivity of substantive effects.

As an illustrative example, consider economic voting: how do economic conditions shape voting behavior? In addition to local GDP growth, growth in neighboring units can matter for evaluations of incumbents through benchmarking effects (Kayser and Peress, 2012). Arel-Bundock, Blais and Dassonneville (2019) demonstrate that many of these theories translate into SLX models: $\theta$, the coefficient on $\mathbf{W} \mathbf{x}$, captures benchmarking effects; $\beta$, the coefficient on $\mathbf{x}$, captures conventional economic voting.

It is difficult to imagine a scenario under which growth in neighboring countries has a larger effect on vote choices than domestic growth, so $|\beta| \geq|\theta|$ seems reasonable. We estimate three models using the Kayser and Peress (2012) data, assuming that their network specification captures the true $\mathrm{W}: 1)$ incumbent vote share regressed on growth (x), plus controls, 2) incumbent vote share regressed on growth $(\mathbf{x})$ and trade-weighted global growth $(\mathbf{W x})$, plus controls; 3) tradeweighted global growth regressed on growth, plus controls. The first model yields a potentially biased estimate $\hat{\beta}(0.530)$, the second model yields estimates for $\beta(0.577)$ and $\theta(-0.173)$ that we treat as 'true', and the third model yields an estimate (0.270) of $\operatorname{Cov}(\mathbf{x}, \mathbf{W x}) / \operatorname{Var}(\mathbf{x})$.

This demonstrates that the main conditions necessary for our bound are plausible (and conservative) in real-world data: $|\theta|=0.173<|\beta|=0.577$, and $\operatorname{Cov}(\mathbf{x}, \mathbf{W x}) / \operatorname{Var}(\mathbf{x})=0.270 \leq 1$. Additionally, the lower bound on $\beta$ estimated from the biased $\hat{\beta}$ - i.e., $\frac{\hat{\beta}}{2}=\frac{0.530}{2}=0.265$ - holds because the bias, in this case, was attenuating. However, this lower bound also holds for alternative W's that we have not considered. That is, we do not need to assume that trade is the appropriate link when making cross-country economic evaluations.

\footnotetext{
${ }^{3}$ The plausibility of $|\beta| \geq|\theta|$ depends on the specific application. We believe it is defensible in most contexts our note addresses: researchers consider spatial effects a nuisance and have little prior information about $\mathbf{W}$.
} 
Similar results follow for the SAR data-generating process,

$$
\mathbf{y}=\alpha+\mathbf{x} \beta+\rho \mathbf{W} \mathbf{y}+\epsilon .
$$

Omitting the spatial lag of the outcome (Wy) induces bias

$$
\operatorname{plim}_{n \rightarrow \infty} \hat{\beta}_{O L S}-\beta=\rho \frac{\operatorname{cov}(\mathbf{W} \mathbf{y}, \mathbf{x})}{\operatorname{var}(\mathbf{x})}
$$

Using condition (3) and the derivation detailed in Betz, Cook and Hollenbach (2019), this expression can be rewritten as

$$
\operatorname{plim}_{n \rightarrow \infty} \hat{\beta}_{O L S}-\beta \leq \frac{\beta \rho}{1-\rho}
$$

with $\hat{\beta}_{O L S}$ in $[0, \infty){ }^{4}$

These results have several implications. First, asymptotically, an omitted spatial lag of the outcome cannot produce a sign reversal on the estimated coefficient. Moreover, the bias is proportional to $\beta$, the true effect.

Second, our assumptions have been purposefully weak. Restricting the domain of $\rho$ yields tighter bounds. For example, with $\rho<0.5$ - which still implies strong spatial interdependence - the bounds on $\beta_{O L S}$ are identical to those derived earlier, $[0,2 \beta]$. Additionally, expression (6) allows for a simple form of sensitivity analysis by determining permissible values of $\rho$ for a desired lower bound on $\beta$, or to graph the lower bound of $\beta$ given $\rho$.

Finally, the bias can again be expressed with data on hand. As we demonstrate in the Appendix, empirical bounds can be calculated from the sample data for arbitrary $\mathbf{W}$, which will be tighter than implied by (6) because they yield a finite upper bound on $\operatorname{cov}(\mathbf{W y}, \mathbf{x})$.

\footnotetext{
${ }^{4} \beta$ in the SAR model reflects the pre-spatial (i.e., partial equillibrium) effect of $x_{i}$ on $y_{i}$. Total effects also involve spatial spillovers and feedback (LeSage and Pace, 2009). The quantity we consider is similar to that obtained by spatial filtering.
} 


\section{Bias from a misspecified network}

Omitting relevant spatial inputs induces bias, yet we can still infer substantively relevant information from such results. Modeling these spatial terms explicitly promises greater gains. To do so, researchers must pre-supply the weights matrix. In applied work, researchers often fear that they do not have sufficient information to accurately specify $\mathbf{W}$, which may cause them to forgo modeling spatial terms at all. Returning to the example of economic voting, Arel-Bundock, Blais and Dassonneville (2019) note that the existing literature provides no theoretically grounded argument for a specific choice of $\mathbf{W}$. Perhaps as a consequence, few studies of conventional economic voting account for benchmarking effects.

Given the centrality of the specification of $\mathbf{W}$, these concerns have received considerable attention (Corrado and Fingleton, 2012; Neumayer and Plümper, 2016). Researchers have suggested

that uncertainty over competing W's can be assessed using information criteria (Halleck Vega and Elhorst, 2015), modeled using Bayesian model averaging (Juhl, N.d.), and may be less essential than presumed because of the high degree of correlation among different W's (LeSage and Pace, 2014). We demonstrate that spatial models with misspecified weights matrices weakly dominate non-spatial models under random measurement error of the weights matrix.

First, consider a SLX process. Suppose instead of $\mathbf{W}$ we possess a noisy $\widetilde{\mathbf{W}}$,

$$
\widetilde{\mathbf{W}} \mathbf{x}=\mathbf{W} \mathbf{x}+\mathbf{e}
$$

where $\mathbf{W} \mathbf{x} \perp \mathbf{e}$, indicating that the spatial lag suffers from classical, non-differential measurement error. Estimating a SLX model yields

$$
\operatorname{plim}_{n \rightarrow \infty} \hat{\theta}_{S L X}=\theta \frac{\sigma_{\mathbf{W} \mathbf{x} \mid \mathbf{x}}^{2}}{\sigma_{\mathbf{W} \mathbf{x} \mid \mathbf{x}}^{2}+\sigma_{\mathbf{e}}^{2}}=\theta \lambda
$$

where $\sigma_{\mathbf{W} \mathbf{x} \mid \mathbf{x}}^{2}$ is the residual variance of regressing $\mathbf{W} \mathbf{x}$ on $\mathbf{x}$, and $\lambda$ is the bivariate reliability ratio (Carroll et al., 2006). Because $\lambda$ is bounded on the unit interval, equation (8) indicates the usual 
attenuation bias.

The corresponding bias in the estimate of $\beta$ is

$$
\operatorname{plim}_{n \rightarrow \infty} \hat{\beta}_{S L X}-\beta=\theta(1-\lambda) \frac{\operatorname{cov}(\mathbf{W} \mathbf{x}, \mathbf{x})}{\operatorname{var}(\mathbf{x})}
$$

This expressions corresponds to the omitted variables bias in equation (2) weighted by $(1-\lambda)$. Thus, the bias in equation (9) can be no greater than the bias in equation (2). Omitting a spatial predictor provides the limit condition of including a spatial predictor with a misspecificed weights matrix. Because $\hat{\beta}_{S L X}$ is less biased than $\hat{\beta}_{O L S}$, the implied lower bound on $\beta$ is also more informative.

For the SAR model, estimation is more complicated: the simultaneity of $\mathbf{y}$ and $\mathbf{W y}$ necessitates maximum likelihood or instrumental variable (IV) methods (Anselin, 1988). While IV strategies typically offer relief from measurement error, this is not the case for spatial models where the instruments are spatially-lagged realizations of the predictors. Because these are generated using the same weights matrix as the outcome, they inherit - and are correlated with - the measurement error. Thus, misspecifying the weights matrix results in asymptotically biased estimates (see the Appendix).

To derive the bias expression in the SAR model, we consider a just identified IV model where $\widetilde{\mathbf{W}} \mathbf{x}$ is used as an instrument for $\widetilde{\mathbf{W}} \mathbf{y}$. Analogously to the SLX model, the IV estimation produces

$$
\operatorname{plim}_{n \rightarrow \infty} \hat{\beta}_{I V}-\beta=\rho(1-\lambda) \frac{\operatorname{cov}(\mathbf{W} \mathbf{y}, \mathbf{x})}{\operatorname{var}(\mathbf{x})}
$$

As before, the bias in equation (10) can be no greater than the bias in equation (6). Consequently, a misspecified weights matrix induces bias in the estimation, but improves over the omitted variable bias from ignoring spatial interdependence.

This should encourage researchers to consider spatial models even where knowledge of the unit ties is imperfect. Not only do spatial estimators of $\beta$ weakly dominate those from non-spatial models, but researchers also obtain sample estimates of $\theta$ or $\rho$. This allows calculating post-spatial and total effects of $\mathrm{x}$ (Franzese and Hays, 2007; LeSage and Pace, 2009), yielding a more complete 
understanding of the relationship of interest.

\section{Simulation}

The following simulations demonstrate the small sample performance of spatial models when W is misspecified. We focus on the SAR model, which is the most widely used spatial model in applied research. ${ }^{5}$ We generate data where both $\mathbf{y}$ and $\mathbf{x}$ are governed by SAR processes:

$$
\begin{aligned}
& \mathbf{y}=\left(\mathbf{I}-\rho_{y} \mathbf{W}\right)^{-1}[\alpha+\beta \mathbf{x}+\boldsymbol{\epsilon}], \\
& \mathbf{x}=\left(\mathbf{I}-\rho_{x} \mathbf{W}\right)^{-1} \mathbf{u},
\end{aligned}
$$

where $\mathbf{u}$ and $\boldsymbol{\epsilon}$ are $N$-length vectors with elements drawn from $\mathcal{N}(0,1) . \beta$ reflects the direct (i.e., pre-spatial) effect of $\mathbf{x}$ on $\mathbf{y}$, while $\rho_{y}$ and $\rho_{x}$ determine the strength of the spatial autocorrelation in $\mathbf{y}$ and $x^{6}$

We hold $\mathbf{W}$ and $\mathbf{u}$ fixed across simulations. Locations for observations are determined by drawing vertical and horizontal coordinates from $\mathcal{U}(0,5)$. Based on these coordinates, we generate a binary 10-nearest-neighbor $\mathbf{W}$ matrix. We fix $\beta$ at 2 and the number of observations to 150 across experiments, focusing on variation in the spatial autoregressive parameters $\rho_{x}$ and $\rho_{y}$, which we vary between 0 (i.e., no spatial interdependence), 0.3 , and 0.6 (i.e., high spatial interdependence). For each of these 9 experimental settings, we simulate 2,000 data sets.

To induce misspecification in the matrix $\widetilde{\mathbf{W}}$ used in the estimation, we generate a second connectivity matrix $(M)$ based on a new random draw of locations. $M$ is therefore independent of the true $\mathbf{W}$ used in the data-generating process. We then generate the set of connectivity matrices used in the model estimation $(\widetilde{\mathbf{W}})$ as a mixture of the true $(\mathbf{W})$ and false $(\mathbf{M})$ matrices. Specifically, the

\footnotetext{
${ }^{5}$ In the Appendix, we present results for a SLX model.

${ }^{6} \mathrm{It}$ is not necessary that the spatial dependence in $\mathrm{x}$ is generated via a spatial autoregressive process. Any alternative which produces spatial correlation in $\mathbf{x}$ (along $\mathbf{W}$ ) would induce the types of biases we consider.
} 
elements $\widetilde{w}_{i, j}$ are determined as

$$
\widetilde{w}_{i, j}= \begin{cases}w_{i, j}, & \text { if } d=0 \text { where } d \sim \operatorname{Bern}(p) \\ m_{i, j}, & \text { otherwise }\end{cases}
$$

where $p$ is the probability of misclassification, which we increase from 0 (no error) to 1 (all error) in increments of 0.05 . In total, this produces 21 connectivity matrices used in estimation, which are all normalized using min-max normalization. ${ }^{7}$

Using the simulated data for $\mathbf{y}$ and $\mathbf{x}$, we estimate a non-spatial linear model (via OLS) and SAR models (via ML) using $\widetilde{\mathbf{W}}$ 's of varying accuracy (decreasing in $p$, the probability of misspecification). For each model, we record $\hat{\beta}$ to assess performance. Figure 1 shows the results for the simulations of the SAR process based on 10-nearest neighbors and min-max normalization. Each cell presents the results for one combination of $\rho_{x}$ and $\rho_{y} ; \rho_{x}$ increases from 0 to 0.6 going from left to right, $\rho_{y}$ increases moving from top to bottom. In each, we plot the densities of coefficient estimates at different levels of the misspecification probability $p$. Darker shading indicates higher levels of misspecification. The densities of $\hat{\beta}$ 's for non-spatial models are plotted in black. The bias in both the non-spatial models and misspecified spatial models increases in $\rho_{y}$ and $\rho_{x}$, being largest in the bottom right cell.

The results underscore three points. First, as the misspecification of $\widetilde{\mathbf{W}}$ increases, the bias in $\hat{\beta}$ increases. Yet even with high interdependence and mismeasured (or omitted) $\mathbf{W}$, the observed bias is much smaller than the bounds derived above. Second, the SAR model weakly dominates the non-spatial model. Even a SAR model estimated with a random $\widetilde{\mathbf{W}}$ does no worse than omitting the spatial term. Finally, the simulation results confirm our analytical results. For example, inequality (6) implies a maximum bias of 3 . The bias in the simulations clearly maintains that bound. Moreover, with $\rho_{y}=\rho_{x}=0.6$, on average we obtain $\frac{\operatorname{cov}(\mathbf{W} \mathbf{y}, \mathbf{x})}{\operatorname{var}(\mathbf{x})}=1.45$. Equation (5) thus implies an OLS estimate of 2.87, identical to the average OLS estimate in the simulations. Tables C. 1 and C. 2 in the Appendix report these quantities for all simulation scenarios.

\footnotetext{
${ }^{7}$ In the Appendix we provide results for row-normalization.
} 


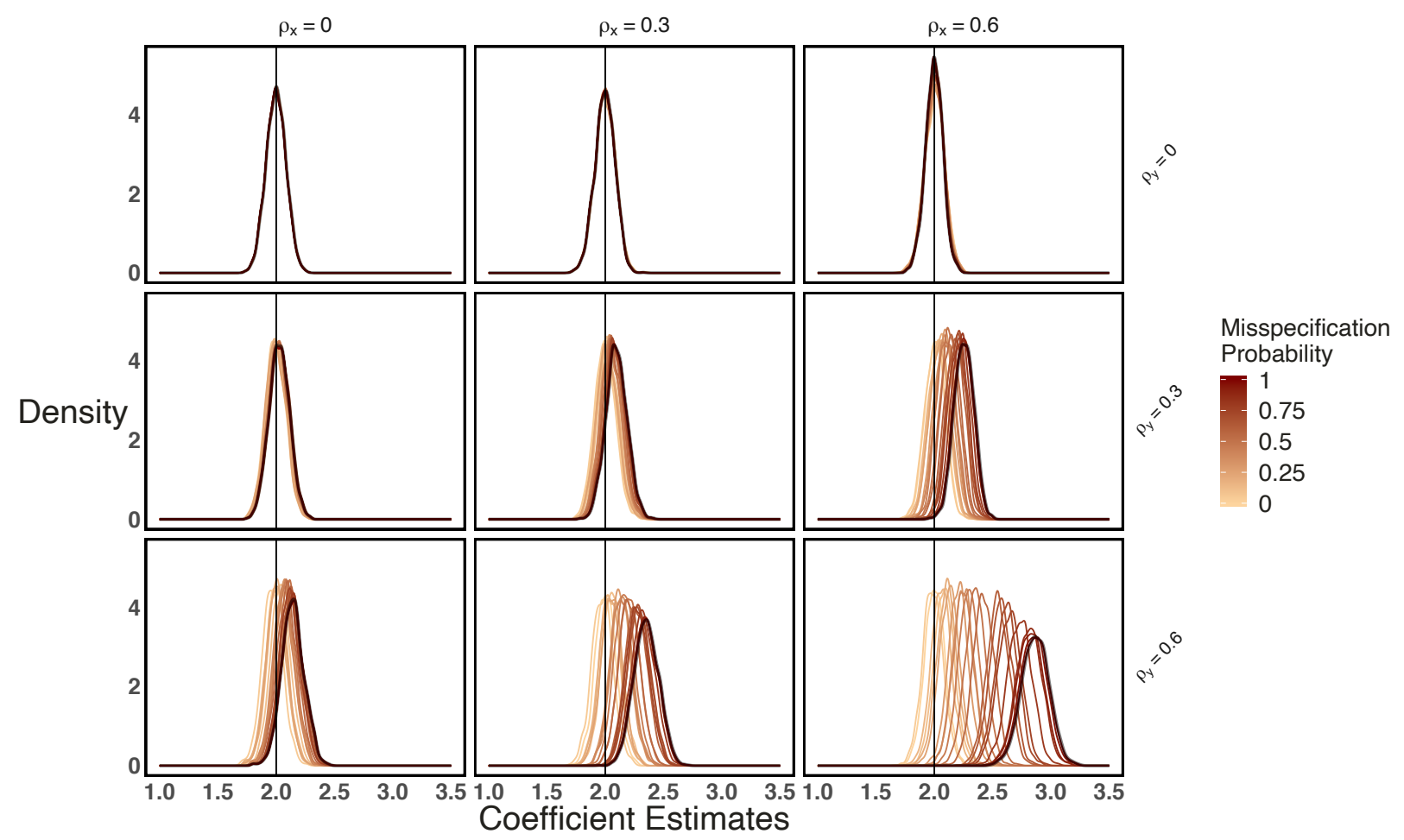

Figure 1: Coefficients with misspecification of W in SAR models based on 10-nearest-neighbors with min-max normalization (colored) and OLS model omitting spatial lag (black).

\section{Conclusion}

Researchers frequently suspect spatial dependence in their data, but lack knowledge of the precise network. Fearing that selecting the wrong network may open them to criticism, researchers may forgo spatial models altogether. Here we have demonstrated the potential biases introduced from omitting spatial terms outright versus including them with error. Our results should encourage the estimation of spatial models even if researchers have imperfect information. As researchers in these settings likely lack strong theory-based specifications, we point to Griffith's five rules of thumb for specifying weights matrices (Griffith, 1996).

We emphasize that our results do not hold under differential measurement error. We suspect that differential measurement error is most likely for network ties that violate the exogeneity assumption for spatial weights - implying that traditional spatial econometric models would be inappropriate. However, we hope that future work extends our results to other contexts and more 
complex forms of measurement error. Several of the features identified here may be useful in these efforts. First, prior research focuses on misspecification in the weights matrix, yet errors manifest in the empirical model as vectors. Second, restrictions on $\mathbf{x}-$ such as limiting the analysis to binary $\mathbf{x}$ - imply restrictions on $\mathbf{W} \mathbf{x}$. Finally, row and min-max normalization imply bounds for the vector range and vector sum. Recognizing these attributes could be of potential use in new analytical and empirical approaches in future research.

\section{References}

Anselin, L. 1988. Spatial Econometrics: Methods and Models. Vol. 4 Springer Science \& Business Media.

Arel-Bundock, Vincent, Andre Blais and Ruth Dassonneville. 2019. "Do Voters Benchmark Economic Performance?" British Journal of Political Science. Advance online publication. doi:10.1017/S0007123418000236.

Betz, Timm, Scott J. Cook and Florian M. Hollenbach. 2019. "Spatial interdependence and instrumental variable models." Political Science Research and Methods. Advance online publication. doi:10.1017/psrm.2018.61.

Carroll, Raymond J, David Ruppert, Leonard A Stefanski and Ciprian M Crainiceanu. 2006. Measurement error in nonlinear models: a modern perspective. Chapman and Hall/CRC.

Cliff, Andrew David and JK Ord. 1981. Spatial Processes Models and Applications. Pion Ltd.

Corrado, Luisa and Bernard Fingleton. 2012. "Where is the economics in spatial econometrics?" Journal of Regional Science 52(2):210-239.

Franzese, Robert J. Jr. and Jude C. Hays. 2007. "Models of Cross-Sectional Interdependence in Political Science Panel and Time-Series-Cross-Section Data." Political Analysis 15(2):140-164.

Griffith, Daniel A. 1996. Some guidelines for specifying the geographic weights matrix contained in spatial statistical models. In Practical handbook of spatial statistics, ed. Sandra L. Arlinghaus. Boca Raton, FL: CRC press pp. 65-82.

Halleck Vega, Solmaria and J Paul Elhorst. 2015. "The SLX model." Journal of Regional Science 55(3):339-363.

Hollenbach, Florian M., Timm Betz and Scott J. Cook. 2019. Replication Data for: Bias due to network misspecification under spatial dependence. Harvard Dataverse.

URL: https://doi.org/10.7910/DVN/UBNPPI

Juhl, Sebastian. N.d. "The Sensitivity of Spatial Regression Models to Network Misspecification." Political Analysis. Forthcoming. 
Kayser, Mark Andreas and Michael Peress. 2012. "Benchmarking across Borders: Electoral Accountability and the Necessity of Comparison." American Political Science Review 106(3):661684.

LeSage, James and R Pace. 2014. "The biggest myth in spatial econometrics." Econometrics 2(4):217-249.

LeSage, James and Robert Kelley Pace. 2009. Introduction to spatial econometrics. Chapman and Hall/CRC.

Neumayer, Eric and Thomas Plümper. 2016. “W.” Political Science Research and Methods 4(1):175-193. 


\section{Online Appendix: Bias due to network misspecification under spatial dependence}

Timm Betz *
Scott J. Cook ${ }^{\dagger}$

\author{
Florian M. Hollenbach
}

*Assistant Professor of Political Science, Department of Political Science, Texas A\&M University, College Station, TX 77843. Email: timm.betz@tamu.edu, URL: www.people.tamu.edu/ timm. betz

${ }^{\dagger}$ Assistant Professor of Political Science, Department of Political Science, Texas A\&M University, College Station, TX 77843. Email: sjcook@tamu.edu, URL: scottjcook.net

${ }^{\ddagger}$ Assistant Professor of Political Science, Department of Political Science, Texas A\&M University, College Station, TX 77843. Email: fhollenbach@tamu.edu, URL: fhollenbach.org 


\section{Appendix A Deriving the Bounds of the Bias in Non-Spatial Models}

In the following we derive the bounds given in Betz, Cook, Hollenbach (n.d.). In keeping with the literature, we assume the weights matrix is a fixed, hollow matrix (no self ties) with exogenously determined non-negative elements. We consider both symmetric and asymmetric spatial weights matrices $\mathbf{W}$ that have been row standardized or scalar normalized using min-max normalization. To ease notation, we assume that $\mathrm{x}$ has mean zero. In detailing our derivation, it is more convenient to re-express the bias expression from the SLX model from the manuscript (equation 2) as ${ }^{1}$

$$
\left(\mathbf{x}^{\prime} \mathbf{x}\right)^{-1} \mathbf{x}^{\prime} \mathbf{W} \mathbf{x} \leq 1
$$

For those familiar with spatial models, the parallel to Moran's I will be obvious, as it is the same ratio used in that measure. There are three virtues of this parallel for our purposes. First, for non-technical readers, it allows us to simplify our bounding condition as being satisfied whenever Moran's I produces values between -1 and 1 , which is generally the case (Cliff and Ord, 1981). ${ }^{2}$ Second, in our technical derivation, we are able to borrow from the literature on Moran's I in detailing the regularity conditions (i.e., assumptions) necessary for this inequality to obtain. Finally, when this condition is not satisfied, it typically also implies that the process is not stationary, meaning the straight-forward application of spatial econometric methods would be ill-advised without additional transformations of the data.

To identify the conditions for inequality (1) to hold, it is useful to distinguish between symmetric and non-symmetric $\mathbf{W}$ matrices. We first show that the condition holds for any arbitrary symmetric $\mathbf{W}$, and then detail sufficient conditions under which it holds for non-symmetric $\mathbf{W}$.

\footnotetext{
${ }^{1} \mathrm{We}$ focus on the case where $\mathbf{x}^{\prime} \mathbf{W x}$ is positive, but the same conditions which ensure inequality (1) to hold also ensure that $\left(\mathbf{x}^{\prime} \mathbf{x}\right)^{-1} \mathbf{x}^{\prime} \mathbf{W} \mathbf{x} \geq-1$.

${ }^{2}$ Moran's I includes a scaling factor as well, which is the sample size $N$ divided by the sum of all elements of W. After row or min-max normalization, this ratio is always larger than one. The sum of all elements of $\mathbf{W}$ is equal to the sum of all row sums as well as to the sum of all column sums. With row-normalization, this sum is identical to $N$; with min-max normalization, it is at most $N$.
} 


\section{Symmetric W}

Our derivation uses that, for any non-zero vector $\mathbf{x}$, the expression $\left(\mathbf{x}^{\prime} \mathbf{x}\right)^{-1} \mathbf{x}^{\prime} \mathbf{W} \mathbf{x}$ takes on values within the field of values (or the numerical range) of the matrix $\mathbf{W}$. For symmetric $\mathbf{W}$, the numerical range is on the real line, with endpoints determined by the largest and the smallest eigenvalues of W. De Jong, Sprenger and Van Veen (1984), for example, use this feature when showing that for symmetric $\mathbf{W},\left(\mathbf{x}^{\prime} \mathbf{x}\right)^{-1} \mathbf{x}^{\prime} \mathbf{W} \mathbf{x}$ lies within the smallest and largest eigenvalue of $\mathbf{W}$. Therefore, applying any familiar normalization strategy (row standardization, min-max, spectral) to a symmetric weights matrix (including common constructions based on contiguity, inverse distance, block group, common border length, fixed buffer) ensures that condition (1) holds, since the maximum eigenvalue of the normalized matrix is 1 (this is also the largest eigenvalue in absolute terms, that is, no eigenvalue is smaller than -1 ; and note that for real symmetric matrices, no eigenvalues are complex).

Using a different approach, we prove this independently by right multiplying and subtracting both sides by $\mathbf{x}^{\prime} \mathbf{x}$. Re-arranging terms this can now be written as:

$$
\mathbf{x}^{\prime}(\mathbf{W}-\mathbf{I}) \mathbf{x} \leq 0
$$

where $\mathbf{I}$ is an identity matrix of size $N$. This expression is now in the more-familiar quadratic form - i.e., $\mathbf{x}^{\prime} \mathbf{A x}$, where $\mathbf{x}$ is a vector and $\mathbf{A}=(\mathbf{W}-\mathbf{I})$ is a symmetric matrix - allowing us to exploit well-known results.

By definition, the expression in (2) is satisfied whenever $\mathbf{A}$ is negative semi-definite. As such, we need only demonstrate the conditions under which $\mathbf{A}=\mathbf{W}-\mathbf{I}$ is negative semi-definite to prove (1). One way to prove that $\mathbf{A}$ is negative semi-definite is to show that all eigenvalues of $\mathbf{A}$ are non-positive or, equivalently, that the largest eigenvalues of W is at most one. From Gershgorin's circle theorem, this condition holds for all $\mathrm{W}$ that have been normalized using min-max or rownormalization. ${ }^{3}$ To see why, note that Gershgorin's circle theorem implies that all eigenvalues $\lambda$ of

\footnotetext{
${ }^{3}$ Note that this is true trivially for spectral normalization, which normalizes $\mathbf{W}$ to ensure its largest eigenvalue is one.
} 
$\mathbf{W}$ are located in discs with origin $w_{i i}$, such that:

$$
\left|\lambda-w_{i i}\right| \leq \sum_{j \neq i}\left|w_{i j}\right|
$$

Since $w_{i i}=0$ for all $i$ and the off-diagonal elements are non-negative, this is equivalent to

$$
|\lambda| \leq \sum_{i} w_{i j}
$$

which implies that the absolute value of the largest eigenvalue of $\mathbf{W}$ is bounded by the largest row-sum. Moreover, because eigenvalues are identical for the transpose of a matrix, Gershgorin's circle theorem implies that all eigenvalues must also be bounded by the largest column-sum. The minimum of the largest row-sum and the largest column-sum therefore provides a bound on the largest eigenvalue of $\mathbf{W}$. More simply, note that for symmetric matrices, $\sum_{j \neq i} w_{i j}=\sum_{i \neq j} w_{i j}$, such that row- and column-sums are identical. For min-max normalization it follows that the largest eigenvalue is bounded by one. ${ }^{4}$ Similarly, for row-normalization, the largest eigenvalue is one, because all row-sums are equal to one (Ord, 1975). This proves that condition (1) holds for any symmetric W. Note that inequality (4) also rules out eigenvalues smaller than -1 , which ensures that the bound in condition (1) also holds when covariance between $\mathbf{W} \mathbf{x}$ and $\mathbf{x}$ is negative.

Before proceeding, we note that symmetric spatial weights matrices are frequently suggested from theoretical models, and commonly used in applied work. They include any matrix that is based on (undirected) attributes of pairs of observations, such as contiguity matrices, inverse distance matrices, matrices based on bilateral trade flows, and matrices based on distance thresholds.

\section{Non-symmetric W}

Extending this approach directly to non-symmetric matrices (as found in network-based ties) proves more challenging. Above we used the fact that negative semi-definite matrices always satisfy (1). For non-symmetric matrices $\mathbf{B}$, the quadratic form is instead given by $\mathbf{x}^{\prime}\left[\frac{\mathbf{B}+\mathbf{B}^{\prime}}{2}\right] \mathbf{x}$,

\footnotetext{
${ }^{4}$ Alternatively, note that because the largest eigenvalue is bounded by the minimum of the largest row-sum and largest column-sum, spectral normalization ensures a smaller normalization factor than min-max normalization.
} 
such that our condition becomes

$$
\mathbf{x}^{\prime}\left[\frac{\mathbf{W}+\mathbf{W}^{\prime}}{2}-\mathbf{I}\right] \mathbf{x} \leq 0
$$

As before, the goal is to identify which W satisfy this condition. Above, the corresponding condition (4) held anytime the row sums or column sums of the non-diagonal elements were one or less, which was guaranteed by min-max and row-normalization. Relying again on Gershgorin's circle theorem, the non-symmetric case instead requires that, for all eigenvalues $\lambda$ of $\frac{\mathbf{W}+\mathbf{W}^{\prime}}{2}$,

$$
|\lambda| \leq \frac{\sum_{j \neq i} w_{i j}+\sum_{i \neq j} w_{i j}}{2} \leq 1
$$

That is, the non-symmetric case requires that the sum of the row and column sums needs to be less than 2 for each unit or that the largest eigenvalue of $\frac{\mathbf{W}+\mathbf{W}^{\prime}}{2}$ is bounded by one. Note that, for nonsymmetric matrices, it is not the case that the eigenvalues of the sum of matrices are identical to the sum of the eigenvalues. Condition (6) therefore does not hold in general after normalization of W. However, it is satisfied in many cases, and in particular for common spatial weights matrices.

First, the above condition holds for all matrices that are doubly-stochastic, such that a unit's column sum equals its row sum, with elements adding up to one. These matrices need not be symmetric, but they ensure that the largest eigenvalue of $\frac{\mathbf{W}+\mathbf{W}^{\prime}}{2}$ is bounded by one, which in turn guarantees that our condition holds. Doubly-stochastic matrices comprise a large number of weights matrices and are commonly used in theoretical work on the properties of spatial econometric estimators. They imply that each element of $\mathbf{W} \mathbf{x}$ is a weighted average of $\mathbf{x}$, where each $\mathbf{x}$ has the same total influence on the network (note that this influence can be distributed arbitrarily across units). Among others, and in addition to all symmetric variants of doubly-stochastic matrices (including inverse distance and contiguity matrices), this applies to many potentially asymmetric weights matrices based on nearest neighbors (LeSage and Pace, 2014). Indeed, the class of matrices that satisfy our bounds is more general than this and includes all line-sum symmetric matrices, such that the sum of elements in each row equals the sum of elements in each column (but row 
sums need not be identical to each other).

Second, a set of possibly asymmetric matrices that satisfies the above conditions are spectral matrices. Min-max, row, and spectral normalization all ensure that the largest eigenvalue of $\mathbf{W}$ is at most one; this holds for arbitrary W. For spectral matrices, the largest eigenvalue is identical to the numerical radius, and normalization thus ensures that $\mathbf{x}^{\prime} \mathbf{W} \mathbf{x} \leq \mathbf{x}^{\prime} \mathbf{x}$. Spectral matrices include all symmetric matrices (providing another approach to prove the above result for symmetric matrices), but they also include a large class of asymmetric matrices (for a characterization, see, e.g., Goldberg and Zwas 1975).

Third, note that for scalar normalizations, condition (6) always holds if we normalize by the maximum row or column sum - that is, the max-max. While not common to the literature, Kelejian and Prucha (2010) emphasize that any matrix norm $\|\mathbf{W}\|$ - e.g., the maximum eigenvalue, the maximum absolute row sum, the maximum absolute column sum, etc. - serves as a useful normalization factor since it bounds the spectral radius. The choice between different norms is theoretically arbitrary, since each is proportionally equivalent.

Fourth, a vast literature addresses the distribution of Moran's I. Cliff and Ord (1981) demonstrate that generally "the upper bound for $|I|$ will be less than unity, although it could exceed unity for an irregular pattern of weights if [observations] with extreme values of $z_{i}$ are heavily weighted." Put differently, to obtain bounds of Moran's I larger than one in absolute value requires not only an unusual composition of $\mathbf{W}$, but that unusual $\mathbf{W}$ must also coincide in predictable ways with the structure of x. In deriving the feasible range for Moran's I for tessellations, Boots and Tiefelsdorf (2000) have shown this rarely occurs. This is because the combinations of a matrix $\mathbf{W}$ and predictor $\mathrm{x}$ are so atypical that they are unlikely to hold in reasonable observational settings. Conversely, if $\mathbf{W}$ is sufficiently dense, these atypical cases cannot arise, because $\mathbf{W}$ effectively averages over $x_{i}$.

Importantly, if Moran's I is bounded by one - and we are outside the realm of 'irregular' cases 
- our condition always holds. To see why, note that Moran's I is defined as

$$
\mathbb{I}=\frac{N}{S} \frac{\operatorname{cov}(\mathbf{W} \mathbf{x}, \mathbf{x})}{\operatorname{var}(\mathbf{x})},
$$

where $N$ is the sample size $N$ as before and $S$ is the sum of all elements of $\mathbf{W}$. For rownormalization and min-max normalization, $S \leq N$ because both normalizations ensure that either each row-sum or each column sum is at most one. Because the sum of all elements of $\mathbf{W}$ is at most the sum of all row-sums or the sum of all column sums, it follows that $S \leq N$. It follows that if Moran's I is bounded by 1 that $\frac{\operatorname{cov}(\mathbf{W} \mathbf{x}, \mathbf{x})}{\operatorname{var}(\mathbf{x})} \leq 1$ must hold as well. In other words, the matrices that spatial econometric models typically envision ensure that our bounds hold.

Finally, and building on this notion of extreme and unusual cases, we can establish bounds for these outlier scenarios. Because these outlier scenarios depend on the specific realizations of $\mathbf{x}$, we can calculate a bound based on the sample. Observe that the worst case for our bounds is a scenario that creates the largest possible value for $\operatorname{cov}(\mathbf{W} \mathbf{x}, \mathbf{x})$. With the mean of $\mathbf{x}$ being zero, this expression is identical to $\frac{1}{N} \sum_{i=1}^{N} x_{i} \sum_{j=1}^{N} w_{i j} x_{j}$ and is maximized if the most extreme values of $x_{i}$ are paired with the largest values that can be produced by $\sum_{j=1}^{N} w_{i j} x_{j}$.

First consider a row-normalized $\mathbf{W}$ or $\mathbf{W}$ such that min-max normalization results in rowsums that are at most one. Note that in both cases, $\mathbf{W}$ preserves the range of $\mathbf{x}$. Then, $\operatorname{cov}(\mathbf{W} \mathbf{x}, \mathbf{x})$ takes its maximum value if $\mathbf{W}$ is such that each observation is exclusively connected to one of the two most extreme cases realized in the sample - i.e., $\max \left\{x_{i}\right\}$ and $\min \left\{x_{i}\right\}$. This corresponds to a weights matrix that has almost all zero elements. We emphasize that these matrices present extreme forms of asymmetry (in particular, after normalization, the largest row-sum is one while the the largest column sum is $N-1$ ), violate standard assumptions about $\mathbf{W}$ (e.g., those presented in Anselin (1988)), and also represent a network with almost no interdependence: the two largest observations on $\mathbf{x}$ determine the values of $\mathbf{W} \mathbf{x}$ of all other observations in the sample, with no observation being exposed to more than one observation, and no path of any length that connects the two most extreme observations. 
To obtain a bound for $\operatorname{cov}(\mathbf{W} \mathbf{x}, \mathbf{x})$, sort $\mathbf{x}$ such that $x_{1} \geq x_{2} \geq x_{3} \ldots \geq x_{N}$. Then, let $k$ such that for $i \leq k, x_{i} \geq 0$ and for $i>k, x_{i}<0$. Then we have that

$$
\begin{aligned}
\operatorname{cov}(\mathbf{W} \mathbf{x}, \mathbf{x}) & =\frac{1}{N} \sum_{i=1}^{N}\left[x_{i} \sum_{j=1}^{N} w_{i j} x_{j}\right] \\
& \leq \frac{1}{N} \sum_{i=1}^{k} x_{i} x_{1}+\frac{1}{N} \sum_{i=k+1}^{N} x_{i} x_{N}
\end{aligned}
$$

which can be calculated in any given sample as an upper bound on $\operatorname{cov}(\mathbf{W x}, \mathbf{x})$. Note that $\sum_{i=1}^{k} x_{i}=-\sum_{i=k+1}^{N} x_{i}$ because $\sum_{i=1}^{N} x_{i}=0$ (which implies that the largest positive covariance is identical to the largest negative covariance, such that we do not have to consider these cases separately).

This bound can be larger than one, but it need not be. For example, it is easy to verify that for any binary $\mathbf{x},{ }^{5}$ this expression simplifies to $\operatorname{cov}(\mathbf{W} \mathbf{x}, \mathbf{x}) \leq 1$. That the bound is always smaller than one for binary $\mathrm{x}$, but not more generally, also reinforces the earlier point: for our main condition to fail, we would need to have a specific constellation of $\mathbf{W}$ and $\mathbf{x}$. Moreover, this bound allows calculating the largest possible value of $\operatorname{cov}(\mathbf{W x}, \mathbf{x})$ in any given sample and, from that, the minimum value of $\rho$ that would be necessary to obtain a bias larger than $\beta$.

To obtain more intuition for the inequality for our worst-case bounds, we can also write

$$
\frac{\operatorname{cov}(\mathbf{W} \mathbf{x}, \mathbf{x})}{\operatorname{var}(\mathbf{x})} \leq\left[\frac{\left(x_{1}-x_{N}\right)^{2}}{4 \operatorname{var}(\mathbf{x})}\right]\left[\frac{\mathbb{E}\left[x_{i} \mid x_{i} \geq 0\right]+\mathbb{E}\left[x_{i} \mid x_{i}<0\right]}{x_{1}-x_{N}}\right]
$$

The first term in this expression is a Popoviciu ratio: it shows how close $\operatorname{var}(\mathbf{x})$ is to its possible maximum based on the largest and smallest value of $\mathbf{x}$. This ratio is at least one and attains the lower bound of 1 if $\mathrm{x}$ is dichotomous. The second term is an indicator of how spread out $\mathrm{x}$ is over its interior. This ratio is at most 1 and attains its upper bound again if $\mathrm{x}$ is dichotomous.

We next consider the case where $\mathbf{W}$ has been normalized with min-max normalization that resulted in column-sums of at most one but potentially larger row-sums (because the largest column-

\footnotetext{
${ }^{5}$ Recall that we assume that $\mathbf{x}$ has mean zero, which implies that a binary $\mathbf{x}$ takes on values $1-p$ and $-p$, where $p$ is the proportion of positive observations in the sample.
} 
sum was smaller than the largest row-sum). The key here is to observe that in this case, each unit has a total influence of at most one on the entire network. Thus, the largest possible value for $\operatorname{cov}(\mathbf{W x}, \mathbf{x})$ is obtained if $\mathbf{W}$ is a matrix that sums all positive values of $\mathbf{x}$ and associates them with $x_{1}$ and that sums all negative values of $\mathbf{x}$ and associates them with $x_{N}$. Put differently,

$$
\begin{aligned}
\operatorname{cov}(\mathbf{W} \mathbf{x}, \mathbf{x}) & =\frac{1}{N} \sum_{i=1}^{N}\left[x_{i} \sum_{j=1}^{N} w_{i j} x_{j}\right] \\
& \leq \frac{1}{N} \sum_{i=1}^{k} x_{i} x_{1}+\frac{1}{N} \sum_{i=k+1}^{N} x_{i} x_{N}
\end{aligned}
$$

which is identical to the expression in (9).

Recall that these bounds would imply that all units are only connected to the most extreme observations. If this were unreasonable, as it often is, tighter bounds would obtain. For example, if one is willing to assume that each unit is connected to a minimum number of observations, tighter bounds can be derived. Consequently, in almost all practical examples the bounds given by (1) will give a more typical approximation than those given by (9) - as we demonstrate using simulations for $k$-nearest neighbors and row standardization in the appendix. However, for completeness, we have presented both sets of bounds here.

\section{SAR: powers of matrices and bounds}

For any (symmetric or non-symmetric) matrix that satisfies $\operatorname{cov}(\mathbf{W} \mathbf{x}, \mathbf{x}) / \operatorname{var}(\mathbf{x}) \leq 1$, it follows that $\operatorname{cov}\left(\mathbf{W}^{k} \mathbf{x}, \mathbf{x}\right) / \operatorname{var}(\mathbf{x}) \leq 1$ for $k=1,2,3, \ldots$. To prove this, note that the maximum of $\operatorname{cov}\left(\mathbf{W}^{k} \mathbf{x}, \mathbf{x}\right) / \operatorname{var}(\mathbf{x})$ is equal to the numerical radius of $\mathbf{W}$. Denoting the numerical radius with $r(\mathbf{W})$, the Halmos inequality establishes that $r\left(\mathbf{W}^{k}\right) \leq r^{k}(\mathbf{W})-$ see, e.g., Goldberg and Zwas (1975). If $r(\mathbf{W}) \leq 1$, it follows that $r\left(\mathbf{W}^{k}\right) \leq r^{k}(\mathbf{W}) \leq 1$, which proves that $\operatorname{cov}\left(\mathbf{W}^{k} \mathbf{x}, \mathbf{x}\right) / \operatorname{var}(\mathbf{x}) \leq 1$ whenever $\operatorname{cov}(\mathbf{W} \mathbf{x}, \mathbf{x}) / \operatorname{var}(\mathbf{x}) \leq 1$

Alternatively, an analogous approach to above can be used to derive bounds from the sample data on $\mathbf{x}$ and $\mathbf{y}$ to calculate a bound on the covariance $\operatorname{cov}(\mathbf{W} \mathbf{y}, \mathbf{x})$ for arbitrary $\mathbf{W}$. For $\mathbf{W}$ such that normalization results in a largest row-sum of at most one, the largest possible value for 
$\operatorname{cov}(\mathbf{W y}, \mathbf{x})$ obtains if $\mathbf{W}$ matches all positive $x_{i}$ with the largest value of $\mathbf{y}$ and all negative values of $x_{i}$ with $y_{\mathrm{T}}=\min \left\{0, \min \left\{y_{i}\right\}\right\}$. Note that if $y_{i}>0$ for all $i$, this implies a matrix $\mathbf{W}$ such that observations with $x_{i}<0$ are 'islands', with only zero elements in the corresponding rows (which is inconsistent with a large class of standard spatial weights matrices and hence results in a bound larger than what those matrices would permit). Then, defining $k$ as before such that $x_{i} \geq 0$ for $i \leq k$ and $x_{i}<0$ for $i>k$,

$$
\operatorname{cov}(\mathbf{W} \mathbf{y}, \mathbf{x})<=\frac{1}{N}\left[\sum_{i=1}^{k} y_{\max } x_{i}+\sum_{i=k+1}^{N} y_{\mathrm{T}} x_{i}\right]
$$

which can be calculated from the data. A similar expression follows for $\mathrm{W}$ if normalization results in a column sum of at most one. The larger of the two expressions can then be used to derive an upper bound for the bias in the SAR case. 


\section{Appendix B Propagation of Measurement Error in the IV Estimation}

To see that the measurement error in $\mathrm{W}$ is not solved by instrumental variable estimation of the SAR model, consider that pre-multiplying both sides of equation (4) by $\mathrm{W}$ and using repeated substitution for $\mathbf{W y}$ yields

$$
\mathbf{W} \mathbf{y}=\beta \mathbf{W} \mathbf{x}+\beta \rho \mathbf{W}^{2} \mathbf{x}+\beta \rho^{2} \mathbf{W}^{3} \mathbf{x}+\ldots+\mathbf{W} \epsilon+\rho \mathbf{W}^{2} \epsilon+\rho^{2} \mathbf{W}^{3} \epsilon \ldots
$$

which demonstrates how $\mathbf{W x}$ and its powers have strength as instruments for $\mathbf{W y}$. However, if we rely on $\widetilde{\mathbf{W}}$ from equation (8) we obtain:

$$
\widetilde{\mathbf{W}} \mathbf{y}=\beta \widetilde{\mathbf{W}} \mathbf{x}+\beta \rho \widetilde{\mathbf{W}^{2}} \mathbf{x}+\beta \rho^{2} \widetilde{\mathbf{W}}^{3} \mathbf{x}+\ldots+\widetilde{\mathbf{W}} \epsilon+\rho \widetilde{\mathbf{W}}^{2} \epsilon+\rho^{2} \widetilde{\mathbf{W}}^{3} \epsilon \ldots
$$

Due to the common transformation via $\widetilde{\mathbf{W}}$, the instrument $\widetilde{\mathbf{W}} \mathbf{x}$ is related to the measurement error in $\widetilde{\mathbf{W}} \mathbf{y}$. IV estimation will resolve the simultaneity bias - the usual concern with spatially-lagged outcomes - but not the bias due to measurement error. 


\section{Appendix C Additional Simulation Results}

\section{C.1 Additional SAR Simulation Results}

In this section, we present results from the additional simulations of the SAR process. ${ }^{6}$ First, Figure C. 1 shows the results from the simulations where $\mathbf{W}$ is based on 10-nearest-neighbors but is row-normalized. Again, spatial dependence in $\mathrm{X}$ increases from left to right, while the spatial parameter of $\mathrm{Y}$ increases from zero for simulation results depicted in the top row to 0.6 in the bottom row. The results are quite similar to those presented in the paper. The biases in the standard linear models and the misspecified SAR models increase with higher spatial dependence in both $\mathrm{X}$ and $\mathrm{Y}$. Again, under all scenarios, the bias misspecified in SAR models is bounded from above by the bias in the non-spatial models.

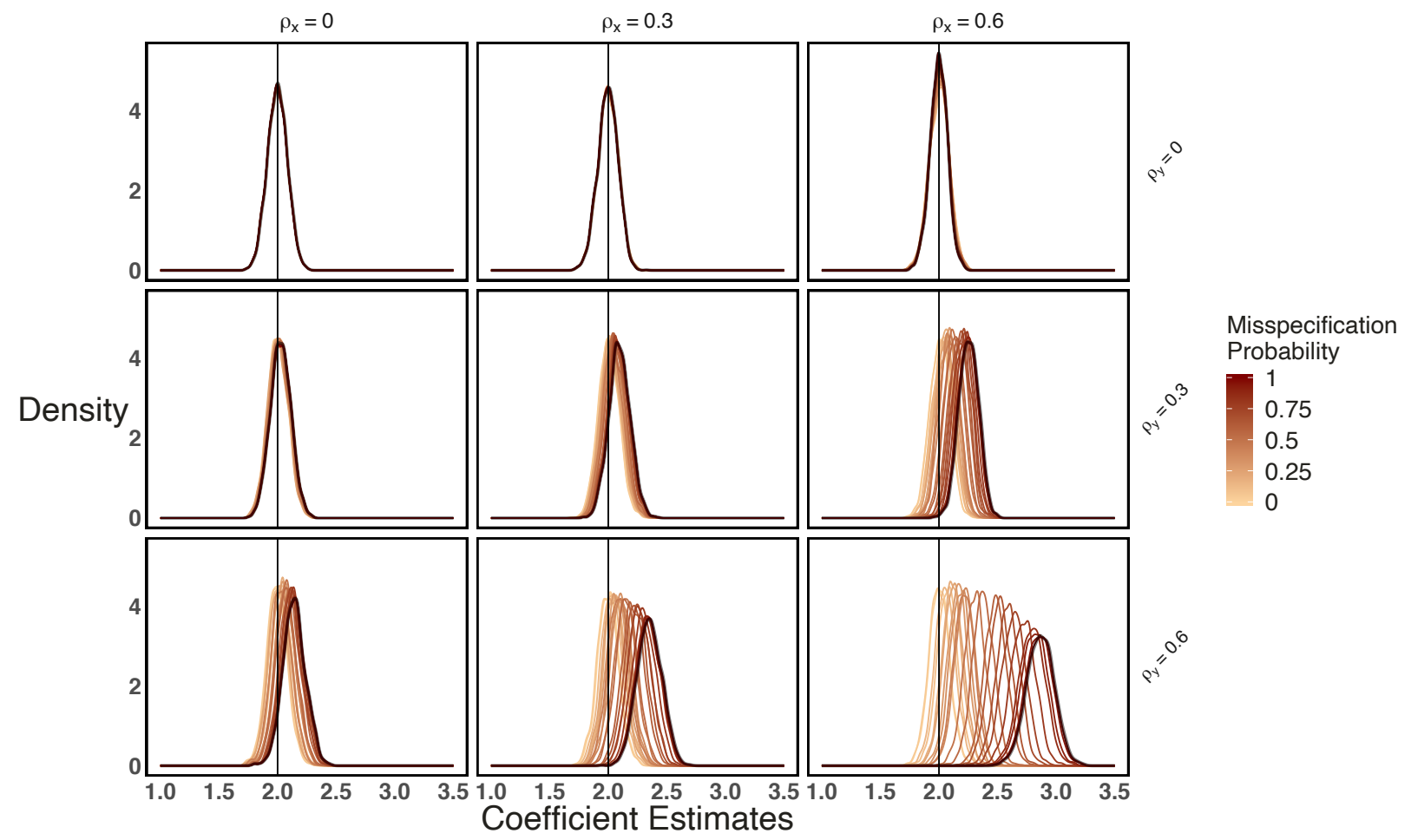

Figure C.1: Misspecification of W in SAR models - KNN \& Row-Normalization

\footnotetext{
${ }^{6}$ The replication materials for the results presented both here and in the main text can be found at Hollenbach, Betz and Cook (2019).
} 
The derivation of our analytical bounds allows us to calculate the expected bias for SAR models. In particular, equation 5 in the manuscript can be rearranged such that we can derive an expected $\hat{\beta}$ given the simulated scenarios: $\operatorname{plim}_{n \rightarrow \infty} \hat{\beta}_{O L S}=\beta+\rho \frac{\operatorname{cov}(\mathbf{W y}, \mathbf{x})}{\operatorname{var}(\mathbf{x})}$.

In Table C.1, we compare the expected $\hat{\beta}$ given our analytical derivation to the average OLS estimate in our simulations given combinations of the spatial parameters in the simulations based on 10-nearest-neighbors and min-max normalization. The first two columns show the variation in the simulated spatial dependence $\left(\rho_{x}\right.$ and $\left.\rho_{y}\right)$, next we calculate the average $\operatorname{cov}(\mathbf{W y}, \mathbf{x})$, and $\operatorname{var}(\mathbf{x})$, and their ratio from the simulations. Based on the ratio $\frac{\operatorname{cov}(\mathbf{W} \mathbf{y}, \mathbf{x})}{\operatorname{var}(\mathbf{x})}$ and true $\beta=2$, we can then calculate the expected $\hat{\beta}$ given the analytical results. In contrast, the last column shows the average $\hat{\beta}$ estimated in the standard linear model at a given scenario. As one can see, the analytical results and the simulated quantities are effectively the same.

Table C.1: Analytical $\hat{\beta} \&$ Mean $\hat{\beta}$ in Simulations - SAR

\begin{tabular}{ccccccc}
\hline$\rho_{x}$ & $\rho_{y}$ & $\operatorname{cov}(\mathbf{W} \mathbf{y}, \mathbf{x})$ & $\operatorname{var}(\mathbf{x})$ & $\frac{\operatorname{cov}(\mathbf{W} \mathbf{y}, \mathbf{x})}{\operatorname{var}(\mathbf{x})}$ & Expected $\hat{\beta}$ & Mean $\hat{\beta}_{O L S}$ \\
\hline 0.00 & 0.00 & 0.00 & 0.91 & 0.00 & 2.00 & 2.00 \\
0.00 & 0.30 & 0.07 & 0.91 & 0.08 & 2.02 & 2.03 \\
0.00 & 0.60 & 0.22 & 0.91 & 0.24 & 2.14 & 2.14 \\
0.30 & 0.00 & 0.17 & 0.94 & 0.18 & 2.00 & 2.00 \\
0.30 & 0.30 & 0.30 & 0.94 & 0.32 & 2.09 & 2.10 \\
0.30 & 0.60 & 0.56 & 0.94 & 0.59 & 2.36 & 2.35 \\
0.60 & 0.00 & 0.69 & 1.18 & 0.58 & 2.00 & 2.00 \\
0.60 & 0.30 & 1.00 & 1.18 & 0.85 & 2.26 & 2.26 \\
0.60 & 0.60 & 1.71 & 1.18 & 1.45 & 2.87 & 2.87 \\
\hline
\end{tabular}

\section{C.2 Additional SLX Simulation Results}

To simulate the SLX models we begin with the following data generating process:

$$
\begin{aligned}
& \mathbf{y}=\alpha+\beta \mathbf{x}+\theta \mathbf{W} \mathbf{x}+\epsilon, \\
& \mathbf{x}=\left(\mathbf{I}-\rho_{x} \mathbf{W}\right)^{-1} u,
\end{aligned}
$$

where $u$ and $\epsilon$ are $\mathcal{N}(0,1)$. The effect paramaters are $\beta, \theta_{x}$, and $\rho_{x}$, with $\beta$ reflecting the direct 
(i.e., pre-spatial) effect of $\mathbf{x}$ on $\mathbf{y}, \theta_{x}$ the spillover effect, and $\rho_{x}$ the spatial interdependence in $\mathbf{x}$. As in the main text, the binary $\mathbf{W}$ matrix is generated with ones assigned to each observation's ten nearest neighbors. We again hold $\mathbf{W}, \beta=2, N=150$, and $u$ fixed across the simulations, focusing on variation in the spatial parameters $\rho_{x}$ and $\theta$. We vary $\rho_{x}$ from 0 (no spatial interdependence) over 0.3 to 0.6 (high spatial interdependence). $\theta$ takes on the following values: 0,1 , and $2 .{ }^{7}$ For each of these 9 experimental settings, we simulate 2, 000 data sets, which leads to 18,000 in total. We again undertake the simulation excercise with $\mathbf{W}$ matrices normalized using two methods: row- and min-max normalization.

Using these simulated data for $\mathbf{y}$ and $\mathbf{x}$, we estimate a non-spatial linear model (via OLS) and the SLX models with the different $\widetilde{\mathbf{W}}$ 's, i.e., the user-specificed weights matrix of varying accuracy (i.e., decreasing in $p$ - the probability of misspecification). We record the estimated $\hat{\beta}$ based on the model's coefficient to assess potential bias.

Figure C.2 shows the results of the SLX simulation analysis for $\mathbf{W}$ based on 10 nearest neighbors and min-max normalization. Each cell in the plot shows the result for one experimental condition, $\rho_{x}$ increases from 0 to 0.6 in cells going from left to right, while $\theta_{x}$ increases in cells moving from top to bottom. Each cell shows the densities of coefficient estimates for models estimated with OLS or SLX models at different levels of the misspecification probability $p$, where darker shading is indicative of higher levels of misspecification. The densities for the OLS models are plotted in black. As one can see, the bias in misspecified models increases in both $\theta$ and $\rho_{x}$, being largest in the bottom right cell. Again, the estimates of SLX models become increasingly worse with higher levels of misspecification, but is bounded from above by the standard linear model estimate.

Figure C. 3 shows the simulation results when the $10-\mathrm{NN}$ matrix is standardized using rownormalization. The results across these specifications are effectively the same as in the SAR process simulations.

Lastly, we again calculate the expected $\hat{\beta}$ based on our analytical derivation for the SLX model

\footnotetext{
${ }^{7}$ The parameter values from $\theta$ are larger than $\rho_{y}$ in the main text because the implied effect on $y$ from changes to $\mathbf{W x}$ are much smaller than from changes to $\mathbf{W y}$.
} 


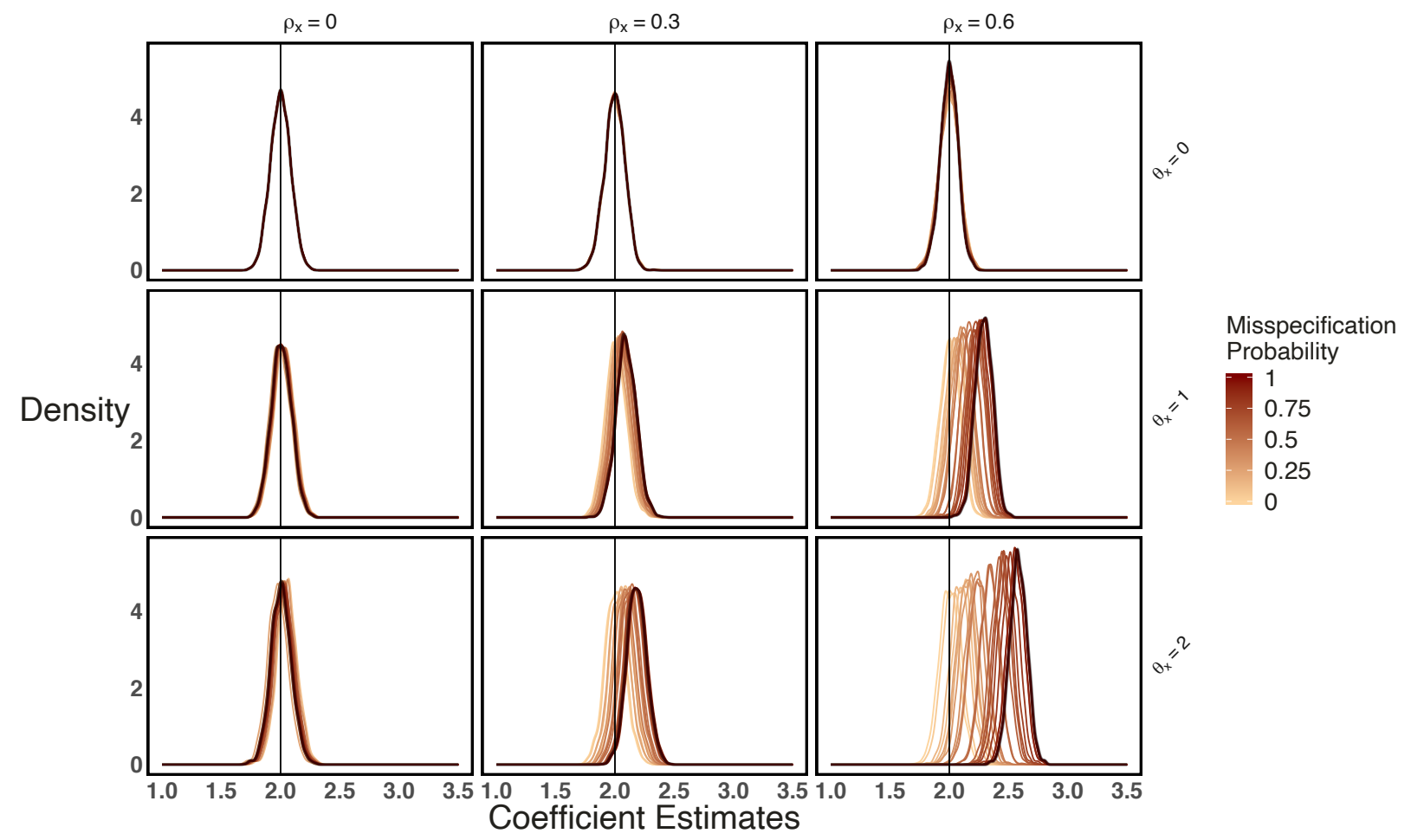

Figure C.2: Misspecification of W in SLX models - KNN \& Min-Max Normalization

(equation 2 in the manuscript) for different scenarios in the simulation with 10 nearest neighbors and min-max normalization. Re-writing equation 2 , we can express the expected estimate from the standard linear model as: $\operatorname{plim}_{n \rightarrow \infty} \hat{\beta}_{O L S}=\beta+\theta \frac{\operatorname{cov}(\mathbf{W} \mathbf{x}, \mathbf{x})}{\operatorname{var}(\mathbf{x})}$. As above, we calculate the expected $\hat{\beta}$ and compare it to the average OLS estimate for each combination of parameters in the simulation. As shown in Table C. 2 the average simulation results for $\hat{\beta}$ are again quite similar to those derived analytically. 


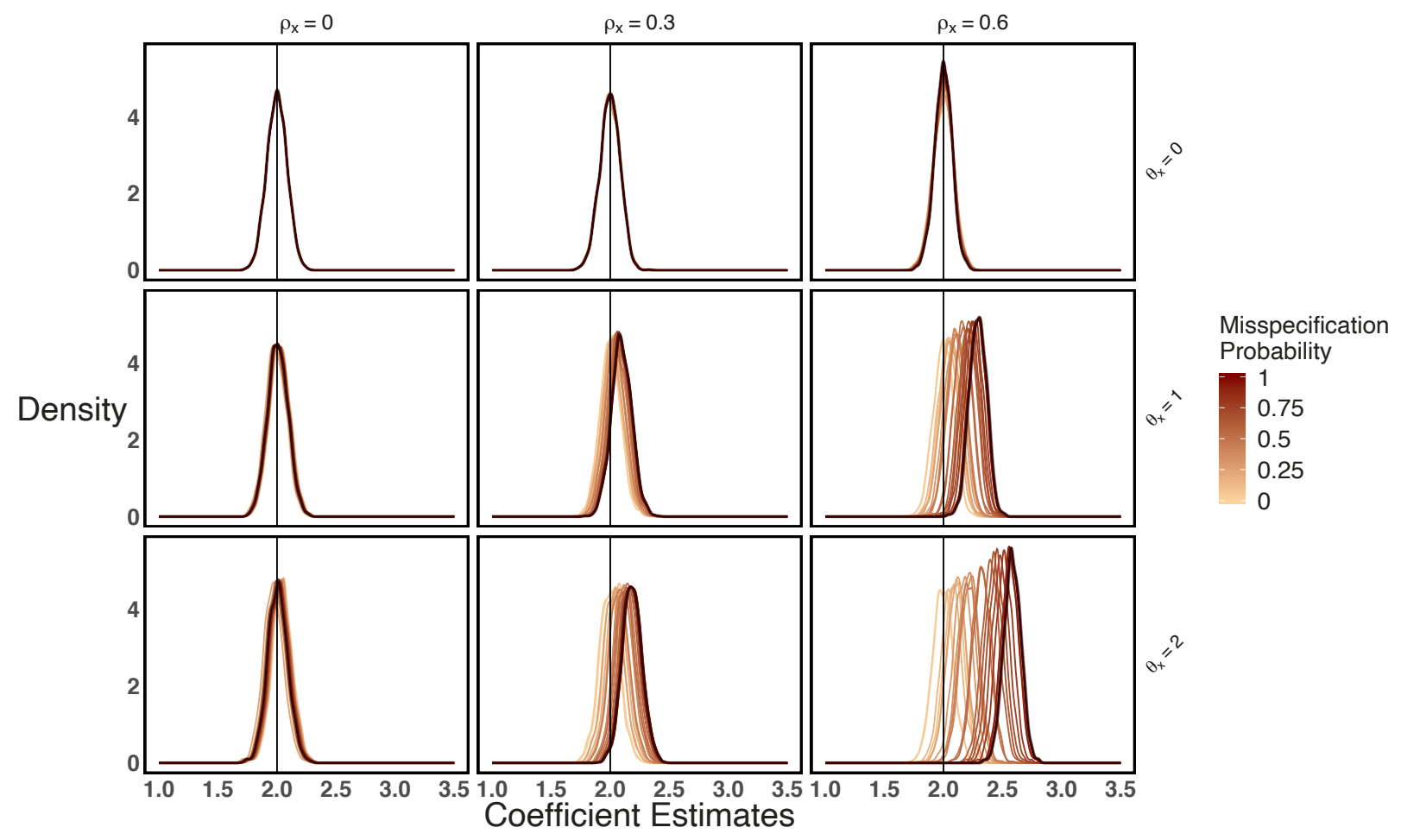

Figure C.3: Misspecification of W in SLX models - KNN \& Row Normalization

Table C.2: Analytical $\hat{\beta} \&$ Mean $\hat{\beta}$ in Simulations - SLX

\begin{tabular}{ccccccc}
\hline$\rho_{x}$ & $\theta$ & $\operatorname{cov}(\mathbf{W} \mathbf{x}, \mathbf{x})$ & $\operatorname{var}(\mathbf{x})$ & $\frac{\operatorname{cov}(\mathbf{W} \mathbf{x}, \mathbf{x})}{\operatorname{var}(\mathbf{x})}$ & $\operatorname{Expected} \hat{\beta}$ & Mean $\hat{\beta}_{O L S}$ \\
\hline 0.00 & 0.00 & 0.00 & 0.91 & 0.00 & 2.00 & 2.00 \\
0.00 & 1.00 & 0.00 & 0.91 & 0.00 & 2.00 & 2.01 \\
0.00 & 2.00 & 0.00 & 0.91 & 0.00 & 2.00 & 2.00 \\
0.30 & 0.00 & 0.09 & 0.94 & 0.09 & 2.00 & 2.00 \\
0.30 & 1.00 & 0.09 & 0.94 & 0.09 & 2.09 & 2.10 \\
0.30 & 2.00 & 0.09 & 0.94 & 0.09 & 2.18 & 2.18 \\
0.60 & 0.00 & 0.34 & 1.18 & 0.29 & 2.00 & 2.00 \\
0.60 & 1.00 & 0.34 & 1.18 & 0.29 & 2.29 & 2.29 \\
0.60 & 2.00 & 0.34 & 1.18 & 0.29 & 2.59 & 2.58 \\
\hline
\end{tabular}




\section{References}

Anselin, L. 1988. Spatial Econometrics: Methods and Models. Vol. 4 Springer Science \& Business Media.

Boots, Barry and Michael Tiefelsdorf. 2000. "Global and local spatial autocorrelation in bounded regular tessellations.” Journal of Geographical Systems 2(4):319-348.

Cliff, Andrew David and JK Ord. 1981. Spatial Processes Models and Applications. Pion Ltd.

De Jong, P, C Sprenger and F Van Veen. 1984. “On extreme values of Moran's I and Geary's c.” Geographical Analysis 16(1):17-24.

Goldberg, Moshe, Tadmor Eitan and Gideon Zwas. 1975. "The Numerical Radius and Spectral Matrices." Linear and Multilinear Algebra 2(4):317-326.

Hollenbach, Florian M., Timm Betz and Scott J. Cook. 2019. Replication Data for: Bias due to network misspecification under spatial dependence. Harvard Dataverse.

URL: https://doi.org/10.7910/DVN/UBNPPI

Kelejian, Harry H. and Ingmar R Prucha. 2010. "Specification and estimation of spatial autoregressive models with autoregressive and heteroskedastic disturbances." Journal of econometrics 157(1):53-67.

LeSage, James and R Pace. 2014. "The biggest myth in spatial econometrics." Econometrics 2(4):217-249.

Ord, Keith. 1975. "Estimation methods for models of spatial interaction.” Journal of the American Statistical Association 70(349):120-126. 